\title{
Fetal Behavior in High-risk Pregnancies Assessed by Two-Dimensional (2D) and Three-Dimensional/Four-Dimensional (3D)/(4D) Ultrasound: A Review
}

\author{
${ }^{1}$ Asim Kurjak, ${ }^{1}$ Ana Tikvica, ${ }^{1}$ Berivoj Miskovic, ${ }^{1}$ Milan Stanojevic, ${ }^{2}$ Badreldeen Ahmed, ${ }^{1}$ Oliver Vasilj \\ ${ }^{1}$ University Department of Obstetrics and Gynecology, University of Zagreb, Sveti Duh Hospital, Croatia \\ ${ }^{2}$ Department of O bstetrics and Gynecology, Fetomaternal Unit, Hamad Medical Corporation, Doha, Q atar
}

\begin{abstract}
Direct assessment of functional development of the fetal central nervous system is not possible, but the assessment of fetal behavior may provide the possibility to distinct between normal and abnormal brain development. Since the ultrasonographic technique allowed the investigation of spontaneous fetal motor activity in utero first studies of spontaneous prenatal movements and fetal behavior were performed and published. 2D ultrasound was considered somewhat subjective method because information needs observer interpretation. The latest development of three-dimensional (3D) and four dimensional (4D) sonography that overcame some of the limitations of 2D methods enable precise study of fetal and even embryonic activity and behavior. In the following text we reviewed the literature on the behavior in the high-risk pregnancies for cerebral palsy assessed by the ultrasonographic techniques.
\end{abstract}

Keywords. Fetal behavior, high-risk pregnancies, cerebral palsy, behavior in fetus with congenital disorders, antenatal behavior screening.

\section{INTRODUCTION}

Fetal behavior could be described as any fetal action or reaction on stimuli that could be observed by the mother or other more objective method as ul trasonography. Through history, maternal registration of fetal movements and obstetrician auscultation of fetal heartbeats in the previous century were the only methods of the follow up of fetal well being in utero. A turning point in the assessment of fetal behavior was the development of real time two-dimensional (2D) ultrasound that enabled the direct visual ization of fetal anatomy and activity. Investigators started to analyze the dynamics of fetal behavior in comparison with morphological studies which led to the conclusion that fetal behavioral patterns directly reflect developmental and maturational processes of fetal central nervous system. Therefore, it was suggested that the assessment of fetal behavior in different periods of gestation may provide the possibility to distinct between normal and abnormal brain development, as well as early diagnose of various structural or functional abnormalities. ${ }^{1}$ However, 2D ultrasound was considered somewhat subjective method because information needs observer interpretation. The latest development of threedimensional (3D) and four dimensional (4D) sonography that overcame some of the limitations of 2D methods enable precise study of fetal and even embryonic activity and behavior. The use of new technologies showed that fetal activity occurs as early as the late embryonic period, which is far earlier that a mother can sense it and from that time we can say that fetal behavior can be defined as any fetal activity observed or recorded with ultrasonographic equipment.

Although more than 100 years of curiosity in fetal behavior and al most three decades of sustained aw areness and research, the study of fetal behavior has achieved widespread acceptance in perinatal medicine. A s it is not yet possible to assess functional development of the CNS directly, it was the window for the investigators to analyze fetal behavior as a measure of neurological maturation including properties of fetal hemodynamics and the muscular system, as well. ${ }^{2}$ Since this technique al lowed the investigation of spontaneous fetal motor activity inuterofirst studies of spontaneous prenatal movements and fetal behavior were performed and published.

In the following text we reviewed the literature on the behavior in the high-risk pregnancies for cerebral palsy that were defined according to our previous study. ${ }^{3,4} \mathrm{~A}$ Ithough we were aware that maj or prenatal pathologies associated with $C P$ are prematurity, antepartum hemorrhage, complications of multiple pregnancy, genetic disorders, intrauterine infection, intrauterine growth restriction, maternal coagulopathies, multiple congenital anomalies, maternal diseases (hypothyroidism, diabetes, drug abuse, severe pre-eclampsia, viral illness) placental pathology, tight nuchal cord, fetal hemorrhage and fetal rhesus disease, we also included prenatal history of 
neuromuscular disease which can adversely affect fetal movements and their inadequate interpretation. ${ }^{5}$

\section{Fetal Behavior in Pregnancies Complicated by Maternal Diabetes Mellitus Type 1}

A mong previously mentioned risk states for disturbed fetal behavior and motility one of the most investigated is maternal diabetes mellitus, mostly type-1. Those pregnancies were shown to be often complicated by early fetal growth delay and congenital malformations that frequently involve the nervous system. Both abnormal embryogenesis and early growth restriction are suggested to shear the common mechanism. One of the first study on that matter was performed in order to determine whether there is a specific delay in the emergence of specific fetal movement patterns, as an expression of the functional motor development of the nervous system in embryos and fetuses of ten women with type- 1 diabetes. ${ }^{6}$ All women had tight metabolic control achieved with continuous subcutaneous insulin infusion which in half patients started before conception. The results showed that there is a delay of 1-2 weeks in almost all but one of the movement patterns emerging in the first 12 weeks of gestation. O nly fetal breathing movements (FBM ) were observed for the first time at the same gestational age as in the control group. If the results were plotted according to crown-rump length the emergence of fetal movement patterns occurred, how ever, almost at the same time as in the control group. It was concluded, that in well controlled diabetic pregnancy there is a delay in functional motor development of the embryonic and fetal nervous system that was shown not to be very specific but mostly dependant on growth delay. On the contrary, breathing movements emerge relatively early as compared to growth. ${ }^{6} \mathrm{~N}$ ext step in the investigation was to study the effects of tightly controlled maternal (type-1) diabetes mellitus on the development of fetal behavioral states. ${ }^{7}$ These states named $1 \mathrm{~F}$ to $4 \mathrm{~F}$ were previously identified as organizational states of fetal eye movements, body movements and fetal heart pattern that could be observed from 38 to 40 weeks of gestation. ${ }^{8} \mathrm{~A} / \mathrm{l}$ the women that participated in the study were treated with continuous subcutaneous insulin infusion (CSII) therapy and were adjusted to an insulin-pump before conception or during early pregnancy. The fetuses were longitudinally studied between 32 and 40 weeks postmenstrual age, at intervals of 2 weeks with $2 \mathrm{hr}$ recordings of fetal heart rate, uterine contractions and of real-time ultrasound scanning for fetal body movements, breathing and eye movements. Four fetal behavioral states were defined by the presence and the combination of following variables: fetal heart rate, body movements and eye movements. They found poorly devel oped state organization exhibited by the near term fetuses of the diabetic group that was related to maternal parity, but not to pre or postconceptional onset of insulin-treatment. The fetuses of nulliparous diabetic women showed more often asynchrony of transition and interruption of periods of concordant association which resulted in significantly higher percentages of 'no-coincidence' and in low incidence of behavioral states as compared with control fetuses of nulliparous women. On the other hand, in the multiparous diabetic cases studied near term the development of fetal behavioral states was normal, although the low number of those cases must be mentioned. Therefore, the one can conclude that, despite tight control of maternal blood glucose levels, the development of behavioral states was disturbed in fetuses of nulliparous diabetic women. The information about the exact blood levels of glucose before conception and in the early pregnancy that w ere later shown to may have influence on the results was not provided. These first studies showed that real-time ultrasound observation of fetal movement patterns can be used to assess the development of the motor component of the nervous system and that a tight metabolic control, achieved with continuous insulin infusion, does not prevent these disturbances in development. 6,8,9

Previously shown a 1-2 weeks delay in the first appearance of all movement patterns which normally emerge during the first 12 weeks of pregnancy was confirmed by the next study by $\mathrm{M}$ ulder and $\mathrm{co}^{10}{ }^{10}$ B reathing movements were again observed for the first time at an earlier age than in the control fetuses. A gain one-hour recordings were made once a week between the 7th and 17th week of gestation in the diabetic women that were being treated with continuous subcutaneous insulin infusion therapy and data were compared to those obtained in uncomplicated pregnancy. The new findings were made when the emergence of frequently occurring movement patterns was plotted against fetal crown-rump length, which is usually smaller in diabetic pregnancy, and there was still a general delay in comparison with the control group. It was concluded that delay in motor development therefore does not run completely parallel with the delay in growth which indicates the possible existence of a specific diabetes-related influence on the functional development of the embryonic and fetal nervous system. Hyperglycemia was considered to be responsible, as the delay in the emergence of fetal general movements was most profound in the women whose periconceptional quality of glucose control was poor. When investigators looked in to the developmental trends in the occurrence of movement patterns in diabetic pregnancies they found the similarity to those in the control fetuses, with the exception of startles. It was found that before the 9th week of gestation, fetal movements occurred less frequently which was related to the quality of maternal glucose control. A fter 12 weeks, the overall incidence was higher than in the control group, due to an increase in the incidence of breathing movements that was generally slower than in the control group. ${ }^{11}$

The generation of body and breathing movements was studied in relation to behavioral states in near-term fetuses and 
newborn infants of women with well-controlled type-1 diabetes. ${ }^{12}$ B efore birth, 2-h recordings were made of fetal heart rate and of fetal body movements, breathing and eye movements while the neonates were studied polygraphical ly during 6 - $h$ for heart rate, body movements, respiration, eye movements and EEG -pattern. The results showed that the generation of body movements was similar in the fetuses of the diabetic and control groups. After birth, the duration per burst was longer in the diabetic group, both in state 1 and state 2, resulting in increased incidences. The incidence of fetal breathing movements was confirmed to be higher and breathing rate was considerably slower in the diabetic group than in control fetuses what indicates al tered regulation of breathing movements in diabetic pregnancy. In the neonates, breathing rate was identical in both groups; it was sl ower in state 1 than in state 2; such a difference was not present before birth, but periodic breathing occurred more often in the infants of diabetic women, especially in state 2. This study showed the continuity of the poor behavioral state regulation from prenatal into postnatal life in the diabetic group which resembled that of more immature fetuses and infants indicating that this cannot be attributed to the instantaneous unfavorable condition, like hyperglycemia before birth. ${ }^{12}$

For further investigation of the impact of early growth delay on subsequent growth (birth weight) and functional development near term (organizational level of fetal behavioral states) the same group investigated 21 and 10 fetuses of diabetic women, respectively. ${ }^{13}$ They found no relationship between the degree of early growth delay and birth weight, while the mean growth delay per fetus in early diabetic pregnancy was negatively correlated with the occurrence of no-coincidence between behavioral state parameters at 36 weeks. Those results have shown that disorders occurring in early life may underlie abnormal functional development in later life, whereas (catch up) growth is mainly determined during the second half of pregnancy. These results corroborate their following findings in the fetuses at 32-38 weeks of gestation in type-1 diabetic pregnancy. ${ }^{14}$ The occurrence of fetal breathing movements was studied in relation to the fetal heart rate patterns (HRPs) $A$ and $\mathrm{B}$ and to Braxton Hicks' contractions by simultaneous $2-\mathrm{h}$ recordings of fetal heart rate and body, eye and breathing movements. During the recordings, breathing activity remained unchanged at all gestational ages studied, in contrast to the gradual decline in fetal breathing movements seen in normal pregnancy. B raxton Hicks's contractions had no effect on FBM , which differs from the specific distribution of FBM during uterine contractions as previously found in uncomplicated pregnancies. They concluded that the (neural) mechanism underlying FBM differs from that in normal pregnancy resulting in breathing movements in late diabetic pregnancy being not influenced by B raxton Hicks' contractions and not showing a clear-cut state-dependency. ${ }^{14}$
To assess the fetal behavioral response to maternal hypoglycemia Reece and cow orkers achieved low glucose plasma levels that were sufficient magnitude to elicit counter regulatory hormones and a symptomatic response by insulin clamp technique. ${ }^{15}$ The fetuses of insulin-dependent diabetic women was studied in the third trimester by monitoring fetal heart rate and recording fetal body and breathing movements and by performing D oppler waveform analysis with real-time ultrasonography. M aternal levels of glucagon, cortisol, epinephrine, and growth hormone were measured at each plasma glucose level. The results showed that mean number of fetal limb and body movements did not changed depending on maternal blood glucose levels. In addition, no significant reductions in fetal breathing movements or heart rate were observed, al though maternal epinephrine and growth hormone levels were significantly increased. These data suggest that fetal well-being remains unaltered in spite of moderate maternal hypoglycemia in diabetic women. ${ }^{15}$

Unlike the previous described methods for observing fetal movements that were used in the clinical practice in the next study the investigators compared third-trimester fetal biophysical activities in normal and well-controlled insulindependent diabetic pregnancies. ${ }^{16}$ Serial bimonthly fetal biophysical studies were performed from 30 to 38 weeks in 18 normal and 18 well-controlled insulin-dependent diabetic pregnancies. E ach study contained 60 minutes of simultaneous ultrasonographic recordings of fetal breathing movements and rates, baseline heart rate, and body movements. Women in the diabetic group maintained good glycemic control and were delivered of normal infants of weights similar to those of nondiabetic gravitas. Their fetuses had higher mean incidences of fetal breathing movement, fetal heart rates, and fetal breathing rates but lower fetal movements and fetal heart rate acceleration counts than did controls throughout the study. Neither shortnor long-term maternal glycemic levels correlated well with fetal biophysical performance. These results confirmed previously reported results that in spite of good maternal glycemic control fetuses of diabetic women behaved differently from those of no diabetic women. As in this study maternal levels of glucose were followed only from the 30th week it is possible that modulation of fetal biophysical activities could be affected by maternal glycemic status before the last trimester. ${ }^{16}$

To assess the effect of type-I diabetes on the quality of general movements (GM s) K ainer and cow orkers analyzed GM s longitudinal ly in 12 human fetuses at two-weekly intervals from 16 weeks until delivery. ${ }^{17} \mathrm{~A} / \mathrm{l}$ fetuses showed normal GM s at 16 weeks, but from 20 weeks onwards until del ivery five fetuses developed abnormal GM s. The diabetes optimal ity-score was significantly lower in the group with abnormal GM s whereas the pregnancy optimality-score did not differ between fetuses 
with normal and abnormal GMs. These results indicate that type-I diabetes can have a negative impact on prenatally observed GMs and that consistently normal GM s indicate normal neurodevel opmental outcome at 10 months. Although the very important observation on the effect of type-I diabetes on the quality of general movements relatively small number of observed fetuses can not be denied.

To synthetize we can say that all mentioned published studies confirmed at least slight impact of maternal diabetes mel litus on fetal and even embryonic movements and behavior. The delay of mergence of spontaneous motility was shown to be independent on growth delay and changes of motility could not be explained by the mother glycemic control. Disturbed organization of behavioral states and general movements in fetuses in pregnancies complicated by the diabetes mellitus demand further investigation by the 3D and 4D technologies that will clarify the neurobehavioral changes in these fetuses.

\section{Fetal Behavior in Pregnancies Complicated by Intrauterine Growth Restriction (IUGR)}

Intrauterine growth restriction is a common clinical problem which is one of the main risk factors of fetal mortality and morbi dity and can have long-term metabolic consequences, such as an increased propensity for some of the most common diseases of adult life like metabolic syndrome, namely obesity, arterial hypertension, hypercholesterolemia, cardiovascular disease and diabetes mellitus type 1 . One of the first study on the impact of growth restriction on the fetal behavior focused on fetal breathing and on the course of behavioral state. ${ }^{18} \mathrm{To}$ achieve that goal $V$ an $V$ liet and his group used real-time ultrasound scanners to detect fetal eye, body, and breathing movements, and the fetal heart rate of 12 growth-retarded fetuses betw een 36 and 40 w eeks of gestation. The mean incidence of fetal breathing was greater during periods of fetal activity (body and eye movements present, greater heart rate variability) than during quiescence (body and eye movements absent, narrowed heart rate variability) at all gestational ages studied in both lowrisk and growth-retarded fetuses. During periods when one of the state variables was in its active condition while the other two were quiet, or the reverse, the incidence of fetal breathing was intermediate between those found when all three state variables were in agreement. A fter behavioral states had developed, at 38 and 40 weeks, the mean incidence of fetal breathing in the low-risk fetuses was greater during active states than during the quiet state. There was no apparent increase in the degree of linkage between fetal breathing and other expressions of fetal activity after the emergence of behavioral states. ${ }^{18}$ In another study by the same group behavioral state observations were carried out on 12 fetuses which subsequently had birthweights bel ow the 10th percentile. ${ }^{19}$ Their gestational ages at the time of study ranged from 32 to 40 weeks. Real- time ul trasound scanning was used to detect fetal body and eye movements, and the fetal heart rate was continuously recorded using a clinical fetal monitor. The appearance of states seemed to be delayed in the grow th-restricted fetuses since states were present in only three of eight growth-restricted fetuses studied at 40 weeks. Also at 40 weeks, the proportion of discordant association of the state variables was increased in the growthrestricted fetuses as compared to the control. There were no consistent differences between the two groups in the occurrence of defined combinations of parameters of the state variables at earlier ages. The results from this study showed that the growthrestricted fetuses have impaired qual ity and quantity of somatic motility in comparison to low risk fetuses of equivalent gestational age. These observations suggest that some aspects of central nervous system function are disturbed in growthretarded fetuses, even in the absence of fetal distress. ${ }^{19}$

Since asymmetrical intrauterine growth restriction occurs earlier than symmetrical or combined one it was important to study the fetal behavior in the group of fetuses that develope growth restriction in earlier gestational age. For that purpose the behavior of 15 asymmetrical intrauterine growth restricted fetuses was compared to that of a control group of heal thy fetuses by simultaneous cardiotocographic and ultrasonographic examinations. ${ }^{20}$ Behavioral states analysis was carried out according to $\mathrm{Nijhuis} \mathrm{et} \mathrm{al}^{8}$ and fetal movements were automatically synchronized with FHR and grouped for each FHRP. There were no statistical differences in the distribution of FHRP between heal thy and IUGR fetuses. On the other hand quantitative differences were found when the movements investigated were related to FHRP. M oreover IUGR fetuses showed a reduction of state $1 F$ (quiet sleep) and an increase of periods of no coincidence between behavioral state variable when compared to the control group fetuses. These findings, therefore suggest the existence of quantitative differences in fetal behavior in asymmetrical IU GR fetuses when compared to heal thy fetuses. ${ }^{20}$ To see wether these differences were caused by the compromised vascularization the degree of vascular peripheral resistance was evaluated by means of pulsed D oppler ultrasonic equipment in the group of asymmetrical growth restricted fetuses and in control group. ${ }^{21} \mathrm{~A}$ Il fetuses underwent simultaneous cardiotocographic and echographic examinations for two consecutive hours at 36-38 weeks of gestation. The distribution of gross fetal body movements, fetal breathing movements and fetal eye movements was analyzed during the different fetal heart rate patterns. Furthermore, the incidence and organization of fetal behavioral states was investigated. Growth restricted fetuses were divided into two groups on the basis of the presence or absence of end diastolic flow in the fetal thoracic descending aorta. The results were in accordance with previous findings that growth restricted fetuses showed a delay in the integration of behavioral patterns and a lower coincidence of behavioral states. These findings are particularly 
evident in the fetuses with a severe increase of peripheral vascular resistance (absence of end diastolic flow in descending aorta) suggesting that a delay in central nervous system devel opment is present in asymmetrical growth retarded fetuses and that there is a possible relationship of this delay to the degree of peripheral vascular resistance.

Since general movements are considered to be important for prediction of fetal neurobehavior next step was to study the effect of severe intrauterine growth restriction on its quality. ${ }^{22}$ The study was performed longitudinally in 17 human fetuses and fetal movements were recorded by means of weekly $1 \mathrm{~h}$ ultrasound and video registrations, following by neurological examinations after birth. No clear effect of uncomplicated intrauterine growth restriction could be detected on the qual ity of general movements, but the quality was disturbed. General movements became slow and small in amplitude in cases where there was a reduction in the amount of amniotic fluid. Parallel to the onset of abnormal fetal heart rate patterns, general movements became poor in repertoire, while they w ere hardly discerni ble after further deterioration of the fetal condition. W ith the exception of 3 infants with cerebral hemorrhages, the quality of general movements observed just before and after birth was identical. In these infants, the quality of general movements as well as the results of the standardized neurological examination tended to normalize at 3 months and 1 year, respectively. This study showed that in contrast to prenatal period uncomplicated IU GR had no marked effect on the quality of general movements or on the results of the neurological examination at the age of 1 year. ${ }^{22}$ In another study by the same group 17 fetuses with intrauterine growth restriction (IU GR), the quantity of general movements and fetal breathing movements were studied both cross-sectionally and longitudinally. ${ }^{23}$ In IU GR fetuses, crosssectional comparisons were made between the quantity of fetal movements and the fetal clinical condition and the quality of general movements. In addition, the quantity of fetal movements in IU GR was compared with that in uncomplicated pregnancies and in pregnancies complicated by premature rupture of the amniotic membranes. In IUGR, the quantity of general movements declined from 25 weeks gestation onwards, whereas the quantity of fetal breathing movements increased. L ongitudinal assessment of these parameters was obtained in four cases and showed a decline of general movements. No relationship between prenatal longitudinal data and neonatal outcome could be observed. The quantity of general movements as well as that of breathing movements was low in IU GR group with abnormal fetal heart rate patterns compared to group with normal parameters. In group with reduced amount of amniotic fluid only the quantity of breathing movements and not of general movements was low. A similar pattern was found in the relation with the quality of general movements observed during fetal deterioration. Cross-sectional analysis of median values (28-31 weeks gestation) did not reveal differences in the quantity of general movements when IUGR, normal pregnancies and premature rupture of the membranes (with or without oligohydramnios) were compared. The quantity of fetal breathing movements was significantly lower in pregnancies complicated by IUGR and by premature rupture of the membranes with oligohydramnios compared to those of normal pregnancies and premature rupture of the membranes without oligohydramnios. In uncomplicated IUGR, the quantity of general movements and breathing movements was in the same range as in normal uncomplicated pregnancies. Similar to the quality of general movements, the quantitative variables were related to the fetal condition. However, in contrast to the qual ity of general movements, the quantity of general movements and breathing movements showed a high inter- and intraindividual variation. Therefore, the results of this study discouraged the use of quantitative aspects of general movements and breathing movements as reliable indicators of the neurological condition in the individual fetus. ${ }^{23}$ On the other hand Ribbert and cow orkers showed that the assessment of fetal activity may be of hel $p$ in fetuses with a marginally reduced $F H R$ variation, in which prolongation of pregnancy is considered desirable to allow further maturation in utero. ${ }^{24}$ In order to determine changes occurring with time they longitudinally studied fetal heart rate variation, general movements, breathing movements and hemodynamics in 19 intrauterine growth restricted fetuses, who eventually were delivered by caesarean section (CS) because of fetal distress. In 14 of 19 fetuses abnormal velocity wave forms were present from the beginning of the study onwards. FHR variation was initially just within or below the norm and fell further during the last 2 days before CS. General movements and breathing movements fell below the normal range later and in a lower rate of occurrence than $\mathrm{FHR}$ variation. FGM showed a more or less consistent fall in time, whereas FBM showed a wide range throughout the period of observation. The poorest outcome occurred in fetuses with reversed enddiastolic velocities and rapid fall in FHR variation. It was concluded that with progressive deterioration of the fetal condition abnormal velocity wave form patterns occur first; FHR variation is reduced subsequently while GM s and FBM are the last to become abnormal. ${ }^{24}$

In another study fetal heart rate (FHR) variation and movements (FA) was investigated in 27 normally grown fetuses and in 18 fetuses with intrauterine growth restriction (IUGR). ${ }^{25}$ The results confirmed previously shown decrease of fetal movements in IU GR fetuses as compared to normally grown fetuses at all gestation times. The investigators reported that IU GR fetuses also spent a significantly lower proportion of time exhibiting high FHR variation at 28-31 weeks. If the fetal movements were compared to FHR one can conclude that more of the IU GR fetuses had abnormalities of movements. Finally, within the IU GR fetuses, those with small head circumferences (less than $3 r d$ centile) had lower movement rates during periods 
of both low and high FHR variation, though this was only statistically significant for periods of low FHR variation. This published report offered the possibility that objective evaluation of fetal behavior could be used in a clinical setting and could provide a more sensitive method of fetal assessment than biophysical profile scores. ${ }^{25}$

A causal relationship with the impairment of fetal oxygenation has been suggested for a reduction in the incidence of fetal movements and in fetal heart rate variation. To test those hypothesis 16 IU GR fetuses and 13 normally grown fetuses were observed during maternal hyperoxygenation that was applied for $40 \mathrm{~min}$ in order to increase fetal $\mathrm{PO}_{2}$ levels. ${ }^{26}$ All IUGR fetuses had abnormal Doppler blood velocity waveforms of the umbilical artery suggesting an impaired uteroplacental exchange. The effect of hyperoxygenation on fetal breathing and body movements and on fetal heart rate was eval uated. In the IU GR fetuses there was a significant increase in fetal breathing and body movements and in heart rate variation during hyperoxygenation as compared to the preceding control period of $40 \mathrm{~min}$. No significant changes in fetal breathing and body movements were found in the normally grown control fetuses. A surprising observation was the increase of the number of heart rate decel erations after discontinuation of the maternal hyperoxygenation. It was concluded that in IU GR fetuses the increase in fetal heart rate variation and the increase in the incidence of breathing and body movements during maternal hyperoxygenation substantiates the relationship between these variables and the oxygenation status of the fetus. ${ }^{26}$

The implementation of 4D sonography was necessary to find out whether the quantity of fetal facial expression and quality of body movements can be used as an additional diagnostic criterion for prenatal brain impairment in fetuses with growth restriction. For that purpose a prospective study was conducted in 50 pregnant women with a growth restricted fetus and in 50 uncomplicated healthy women in the third trimester of pregnancy. ${ }^{27} 4 \mathrm{D}$ ultrasound observation was specially designed to assess whether functional brain impairment and fetal growth restriction had prenatal ly occurred by the utilization of several behavioral patterns. The results showed that the median value of all movement patterns in the normal fetuses differed from fetuses with intrauterine growth restriction (IU GR). Statistical evaluation reveal ed significant differences in the distribution of the movements between these groups. A tendency that IU GR fetuses have less behavioral activity than normal fetuses was noted in all observed movement patterns. Correlation reached statistical significance between normal and IUGR fetuses in the third trimester in hand to head, hand to face and head retroflexion. Statistically significant differences could be shown in the distribution of the median values of observation over the five qual itative categories of head and hand movements. These recent data on IUGR fetuses obtained by 4D sonography are stimulating and might result in a more effective strategy to assess development before birth and may encourage future use of 4D ultrasound for quantitative and qual itative assessment of fetal behavior as possible indicators of the neurological condition in IU GR fetuses. ${ }^{27}$

\section{Fetal Behavior in Pregnancies Complicated by Alcohol use in Pregnancy}

Alcohol (ethanol) use during pregnancy is important factor which can produce a wide spectrum of effects in the devel oping embryo and fetus that are dependent on the maternal drinking pattern. The effects of chronic ethanol exposure on the developing conceptus includes ethanol teratogenesis, manifesting in the human as the fetal alcohol syndrome and other al cohol effects while acute ethanol exposure on the nearterm fetus include suppressed fetal breathing movements, electrocorticographic activity and electrooculographic activity. ${ }^{28}$ One of the first studies on the effect of maternal ingestion of ethanol was conducted in 11 healthy pregnant women at 37 to 40 weeks' gestation and fetal breathing movements, gross fetal body movements, and fetal heart rate were analyzed. ${ }^{29}$ It was shown that fetal breathing movements were al most abolished within 30 minutes of the al coholic drink and remained significantly decreased for 3 hours which confirmed some previous results. ${ }^{30}$ On the other hand the incidence of gross fetal body movements before or after ethanol was not different from that on the control day, and the fetal heart rate was not changed after maternal ingestion of ethanol.

Disturbed behavioral state organization has been found in the near term fetuses from pregnancies with maternal alcohol abuse. $^{31}$ The abnormalities included frequent interruptions of the periods of concordant association of $2 \mathrm{~F}$-parameters, reflected by a high proportion of no-coincidence, and spontaneous awakenings (State $4 \mathrm{~F}$ ), always following stable periods of State $1 \mathrm{~F}$. The latter phenomenon was not found previously, neither in normal nor in complicated pregnancies. A fter birth normal state organization was found. It was suggested that the abnormal ities in fetal behavior might have been due to maternal alcohol abuse, whereas a possible withdrawal effect might have occurred in utero. ${ }^{31}$

Although disturbed sleep regulation has been observed in neonates of women who drank heavily during pregnancy it was unknown if an occasional drink affects fetal sleeping behavior. For that purpose $\mathrm{M}$ ulder and his group examined the effects on fetal behavioral state organization of two glasses of wine in 28 near-term pregnant women. ${ }^{32}$ Simultaneous 2 -h recordings of fetal heart rate and body, eye, and breathing movements were made on two successive days, once without alcohol exposure and once during maternal alcohol consumption. The study was standardized for time of day and fetal sleep state, i.e. the start of recording was either during quiet sleep or during active sleep. It was found that alcohol intake reduced fetal eye movements, 
disorganized behavioral state organization (rapid eye movement sleep was affected in particular), and suppressed fetal breathing activity al most completely. This study revealed that even modest maternal alcohol intake affected fetal behavioral state organization, which reflects an immediate effect on fetal brain function. ${ }^{32}$

\section{Fetal Behavior in Pregnancies Complicated by Drug use in Pregnancy}

In utero cocaine exposure is associated with poor head grow th, abnormal neurodevelopment, and an increased incidence of sudden, unexplained death, suggesting that in utero cocaine exposure disrupts the central regulation of breathing what is due to altered CNS maturation. Since components of fetal behavioral state organization reflect the successful integration of the central nervous system with a specific developmental timetable, and can be studied by fetal ultrasound techniques, the group of authors developed a strategy for assessing the state organization of the fetus exposed to cocaine in utero by $2 \mathrm{D}$ ultrasound techniques. ${ }^{33} \mathrm{Fetal}$ assessments were accomplished by serial ultrasonographic examination, videotaped, and scored by a scheme developed by the authors. A bnormal or delayed state behavior was identified in 13 of 20 fetuses. State organization was evaluated as suspect or abnormal for 16 of the 20 exposed newborns suggesting that disorganized behavioral state in the fetus successfully predicted abnormal newborn behavior. These findings support the concepts that cocaine exposure disrupts central nervous system development and that fetal assessment of state is predictive of neonatal outcome. ${ }^{33}$

The negative impact of cocaine was report on a series of ultrasound observations on a fetus of the mother who was a user of cocaine until 18 weeks of gestational age, but after this age used it only once at 31 weeks of gestation. ${ }^{34}$ It was shown that exposure to cocaine resulted in prolonged periods of inactivity interspersed with periods of increased general activity and an increased number of startles. This was particularly evident immediately foll owing exposure to cocai ne but behavior appeared to become more normal with time as the mother stopped using cocaine. The behavioral states of the cocaine exposed fetus were also different from unexposed fetuses. L onger periods were spent in states $1 \mathrm{~F}$ and $4 \mathrm{~F}$ and less time in state $2 \mathrm{~F}$. Other studies are in agreement with these findings with additional result of fetal ( $T$ able 1 ) being less time in state $4 \mathrm{~F} .{ }^{35} \mathrm{~F}$ urthermore the incidence of periods where no states were observed was considerably higher. These observations confirmed that maternal cocaine use disrupts the behavior of the fetus indicating a direct effect on the CNS and showed the value of prenatal behavioral observations to assess the effects of neurotoxic agents.
The effect of methadone on fetal neurobehavioral functions was investigated in women attending a substance abuse treatment facility with otherwise uncomplicated pregnancies. ${ }^{36}$ Fetal behavior was evaluated at peak and trough methadone levels and the fetal measures included heart rate, variability, periodic accelerations/decelerations, motor activity, and fetal movement-heart rate coupling. The results reveal ed that fetuses displayed less motor activity, and the integration between heart rate and motor activity was attenuated at peak level of methadone and it was concluded that maternal methadone administration has significant effects on fetal behavioral functions that are independent of maternal effects. ${ }^{36}$

\section{Fetal Behavior in Pregnancies Complicated by other Risk Factors in Pregnancy}

Intrauterine adequate levels of thyroid hormones have been shown to be absolutely necessary for early brain development. During pregnancy, both maternal and fetal thyroid hormones contribute to fetal brain development and maternal supply explains why most of the athyreotic newborns usually do not show any signs of hypothyroidism at birth, while thyrotoxic fetuses may devel op goitre, tachycardia, hydrops associated with heart failure, growth retardation, craniosynostosis, increased fetal motility and accelerated bone maturation. ${ }^{37}$ It was shown that fetal movements and fetal heart rate are the most valuable indicators of thyroid function in the fetus and their intense control is necessary from the beginning of the second trimester in cases of hyperthyroidism during pregnancy. ${ }^{38}$

Fetal body movements and breathing movements as a variables of fetal biophysical profile have been shown to be predictable of impending fetal infection in patients with premature rupture of the membranes. ${ }^{39}$ Some viral and bacterial pathogenes have been shown to influence fetal motility. For example, transplacental infection with coxsackie $B_{3}$ confirmed by molecular techniques resulted in severe reduction of fetal movements at the 27 th week detected by prenatal $2 \mathrm{D}$ ultrasound. Late onset of fetal akinesia deformation sequence with mild arthrogryposis was the finding at fetal autopsy following interruption of the pregnancy. ${ }^{40}$ Listeria monocytogenes has been increasingly recognized as a cause of intrauterine sepsis with associated perinatal wastage. The most common presentations in pregnancy include premature labor, an influenza-like illness and reduced fetal movements. ${ }^{41}$

Intra-amniotic infections often result in preterm premature rupture of membranes. In a prospective study fetal behavior was observed in 41 cases complicated with preterm premature rupture of membranes. ${ }^{42}$ The length of time and the number of fetal breathing and gross body movements were correl ated with the amniotic fluid culture results. A $n$ episode of fetal activity 
Table 1: Additional findings of fetal behavior in high risk pregnancies in published reports

\begin{tabular}{|c|c|c|}
\hline Author & Year & Main findings \\
\hline V isser et al ${ }^{4}$ & 1985 & First study on diabetes-related influence on delay emergence of fetal behavioral pattern \\
\hline Mulder et al ${ }^{5}$ & 1987 & Study on diabetes-related influence on deley emergence of fetal behavioral states \\
\hline Visser et $\mathrm{al}^{7}$ & 1986 & Integrated results on diabetes-related influence on of fetal behavioral pattern trough the gestation \\
\hline Mulder et $a l^{8,9}$ & 1991 & A dditional findings on diabetes-related influence on fetal behavioral pattern \\
\hline Mulder et al ${ }^{10}$ & 1990 & Study on diabetes-related influence on behavioral states in the near-term fetuses \\
\hline Mulder et al ${ }^{11}$ & 1992 & $\begin{array}{l}\text { Indicated that diabetic disorders occurring in early life may influence abnormal functional development in later } \\
\text { gestation }\end{array}$ \\
\hline Mulder et al ${ }^{12}$ & 1995 & Study on fetal breathing movements in relation to other parameters of fetal well being in late diabetic pregnancies \\
\hline Reece et $\mathrm{al}^{13}$ & 1995 & Findings on how maternal hypoglycemia affects fetal behavioral parameters \\
\hline Devoe et al ${ }^{14}$ & 1995 & Study on diabetes-related influence on Fetal biophysical activities in third-trimester \\
\hline Van Vliet ${ }^{15}$ & 1985 & Results on relationship between fetal activity and behavioral states in third trimester growth-restricted fetuses \\
\hline Van V liet ${ }^{16}$ & 1985 & Showed that the quality and quantity of the growth-restricted fetal motility is disturbed \\
\hline Arduini ${ }^{17}$ & 1988 & Showed that the quality and quantity of the assymetrical growth-restricted fetal motility is disturbed \\
\hline Rizzo ${ }^{18}$ & 1987 & Findings on the influence of fetal blood flow on the IUGR fetal motility \\
\hline Sival 19,20 & 1992 & Findings on the IUGR influence on the fetal general movements \\
\hline Ribbert et al ${ }^{21}$ & 1993 & $\begin{array}{l}\text { Showed the dynamic of fetal movements and relations to other parameters of fetal well-being in growth-restricted } \\
\text { fetuses }\end{array}$ \\
\hline Vindla et al ${ }^{22}$ & 1997 & Findings on fetal movements as the predictor of fetal condition \\
\hline B ekedam et al ${ }^{23}$ & 1991 & $\begin{array}{l}\text { The effects of maternal hyperoxia on fetal breathing movements, body movements and heart rate variation in } \\
\text { IU GR fetuses }\end{array}$ \\
\hline A ndonotopo et $a^{24}$ & 2006 & 4D findings on fetal behavior of growth restricted fetuses. \\
\hline McL eod et $\mathrm{al}^{26}$ & 1998 & First study on the effect of maternal ethanol Consumption on fetal body movements \\
\hline Mulder et al 28,29 & 1998 & Revealed that even modest maternal alcohol intake affects fetal behavioral state organization \\
\hline Hume et $a^{30}$ & 1989 & Showed that $\mathrm{n}$ utero cocaine exposure affects fetal behavioral state \\
\hline Hepper et al $\left.\right|^{31}$ & 1995 & A longitudinal study on the effect of maternal cocaine consumption on fetal behavior \\
\hline Jansson et al 33 & 2005 & Revealed the fetal behavior response to maternal methadone exposure \\
\hline Konstantinidou et $\mathrm{al}^{37}$ & 2007 & Case report on the effect of Coxsackievirus $B_{3}$ on fetal motility \\
\hline Craig et $\mathrm{al}^{38}$ & 1996 & Study on the effect of Listeria monocytogenes on fetal motility \\
\hline Goldstein et al ${ }^{39}$ & 1988 & Findings on fetal body and breathing movements as Predictors of intraamniotic infections \\
\hline Del V alle et al ${ }^{40}$ & 1992 & Findings on fetal body and breathing movements as Poor predictors of intraamniotic infections \\
\hline Sherer et al ${ }^{41}$ & 1996 & Showed that decreased amniotic fluid volume before third trimester is associated with decreased fetal motility \\
\hline Habek $D^{42}$ & 2007 & Revealed the effect of chronic tobacco hypoxia on the fetal behavior in early pregnancy \\
\hline Cowperthwaite et al ${ }^{43}$ & 2007 & $\begin{array}{l}\text { Showed that fetuses less than } 37 \text { weeks GA of mothers who smoke throughout pregnancy have a delayed onset of } \\
\text { response to the maternal voice. }\end{array}$ \\
\hline W arner et $\mathrm{al}^{44}$ & 2002 & Study on influence of mothers hypertension on fetal behavior \\
\hline K silevsky et al ${ }^{45}$ & 2005 & Comparison of fetal behavior in low- and high-risk Pregnancies \\
\hline de $V$ ries et al ${ }^{46}$ & 2007 & Overview on changes in fetal motility indicative of malfunction of the central nervous system in high-risk \\
\hline M orokuma et al ${ }^{48}$ & 2007 & First simplified ultrasound screening for fetal brain function based on behavioral pattern. \\
\hline A hmed et al ${ }^{50}$ & 2005 & Provided more information of specific movement patterns and quality of movement in the high risk fetuses. \\
\hline A ndonotopo et $a^{51}$ & 2005 & Allowed early diagnosis of a functionally affected anencephalic fetus. \\
\hline Kurjak et $\mathrm{al}^{52}$ & 2007 & New scoring system for fetal neurobehavior assessd By 3D and 4D US \\
\hline
\end{tabular}


(body movements and breathing movements) of greater than or equal to 30 seconds during 30 minutes of observation was associated with the absence of intraamniotic infection in 100\% of the cases. On the other hand, the absence of fetal breathing movements and gross body movements of less than a 50 second duration during 30 minutes of observation was associated with positive amniotic fluid cultures in all cases. If an episode of fetal breathing movements was present but lasted less than 30 seconds and/or the total time of gross body movements was greater than 50 seconds, $64 \%$ of patients had an intra-amniotic infection. The conclusion was that the breathing movements could be used as a predictor of intra-amniotic infection. On the contrary, study by D el $V$ alle and his group which enrolled sixtyeight women with the diagnosis of preterm premature rupture of membranes (PROM ) showed that the biophysical profile with breathing movements included and the NST are poor predictors of perinatal infectious complications. ${ }^{43}$

To investigate the relation between amniotic fluid volume (A FV) and fetal movements at less than 32 weeks of gestation as assessed by routine biophysical profile (BPP) the study included 352 consecutive nonhypertensive, nondiabetic patients. ${ }^{44}$ The results reveal ed a significant correlation between decreased AFV and decreased fetal movements. Fetal presentation and GA were not significantly different between patients based on score of fetal movements. The authors concluded that decreased AFV is associated with decreased fetal movements irrespective of fetal presentation or gestational age. $^{44}$

Nicotine was shown to have impact on fetal motility in recent published study on fetal movement in early pregnancy that were qualitatively and quantitatively observed by ultrasound monitoring in women smokers and in controls. ${ }^{45}$ The study included three groups of 20 pregnant women each: nonsmokers (group 1), smokers of an average of 10 cigarettes daily (group 2 ), and smokers of an average of $>20$ cigarettes daily (chronic smokers; group 3). T wo-dimensional US study was performed once during gestational weeks $10-20$ by the then standard method of fetal movement monitoring:

1. Quantitative measurement of global fetal movements qualitatively verified as brisk or sluggish

2. Quantitative measurement of isolated spontaneous head movements

3. Quantitative measurement of isolated spontaneous arm movements

4. Quantitative measurement of isolated spontaneous leg movements

5. M-mode measurement of fetal heart rate.

The results showed that the ratio of brisk to sluggish fetal movements was higher in nonsmokers compared to smokers. The rate of isolated spontaneous head and arm movements and of the upper cerebral pattern (head and arm movements) was statistically significantly lower in group 3 as compared with groups 1 and 2, whereas no statistical significance was recorded in isolated spontaneous leg movements. The rate of fetal tachycardia was also significant in group 3, whereas tachyrhythmia was recorded in seven children born to group 3 mothers. The described study of the effect of chronic tobacco hypoxia on the components of fetal behavior reveal ed a positive correlation between global and isolated fetal hypokinesia of the upper cerebral pattern, fetal tachycardia, and tachyrhythmia in the group of mothers who were chronic smokers. ${ }^{45}$

To determine the effects of maternal smoking on fetal spontaneous behavior and auditory processing Cooperwhite and coworkers examined 38 fetuses of smoking and nonsmoking mothers, stratified by gestational age and observed at least $1 \mathrm{hr}$ following smoking. ${ }^{46}$ Observations included spontaneous fetal heart rate and body movements ( $20 \mathrm{~min})$ followed by a $2 \mathrm{~min}$ audiotape of the mother reading a story while FHR and body movements were recorded. They found that the fetuses less than 37 weeks GA of mothers who smoke throughout pregnancy have a delayed onset of response to the maternal voice, a subtle difference which may have implications for later language development for prematurely born infants.

The relationship between maternal blood pressure (BP) and fetal behavior were examined in hypertensive compared to normotensive women at 33 and 36 weeks gestational age. ${ }^{44} \mathrm{As}$ expected, maternal BP was negatively related to $\mathrm{GA}$ at birth and birth weight. ${ }^{47} \mathrm{O}$ average, fetuses of hypertensive women were born 2 weeks earlier ( 38 weeks GA) and 340 gm lighter. $M$ aternal systolic BP was negatively related to the number of spontaneous body movements observed on ul trasound scan over $20 \mathrm{~min}$ and the magnitude of the fetal heart rate acceleration elicited by a vibroacoustic stimulus. A t 36 weeks GA, vibroacoustic stimulation elicited differential responding with fetuses in the hypertensive compared to the normotensive group having fewer body movements, a lower magnitude of FHR acceleration, and a lack of cardiac-body movement coupled responses. These findings suggest a relationship between maternal BP and fetal behavior and differential functional development of sensory-motor response systems which need to be characterized in the subgroups of hypertensive disorders observed during pregnancy.

Recent meta-analyses were conducted on archival data of human fetal behavior to identify differential behavior among high-risk fetuses in pregnancies complicated by threatened preterm delivery, maternal hypertension or diabetes compared with low-risk fetuses in uneventful pregnancies, delivering as healthy, full-term infants. ${ }^{48}$ There were no differences in spontaneous behaviors when scored using clinical criteria for the nonstress test and biophysical profile; how ever, there were differences in the magnitude of the behaviors measured in the tests. Developmental differences were observed between those threatening to deliver early and the fetuses of hypertensive and diabetic mothers. The later two groups differed little from one 
another but differed from low-risk fetuses in their response to auditory stimulation. It was concluded that differences in behavior among high-risk groups suggest that atypical fetal behaviors may represent adaptation to condition specific insult rather than a generalized response to insult per se The finding that high-risk fetuses showed atypical responses to auditory stimuli indicates a need to examine the relation between fetal auditory function and later language acquisition.

\section{Behavior in Fetuses with Congenital Disorders}

A lthough behavior of fetuses with congenital disorders has been investigated and published in many, mostly case-reports publications systematic approach to the issue lacks mostly because high diversity of disturbances. A lthough estimation of neurodevelopmental outcome of the fetuses based on the following studies could be possible, a direct precise prognosis is still not available during the prenatal period. The use of $4 D$ US recording could provide more information about the qual ity of fetal movements leading to the better prediction of neuronal development.

$D e V$ ries conducted a literature search on motility in fetuses with congenital disorders. The review included 48 articles describing motility of 104 fetuses. ${ }^{49}$ The author divided abnormal motility into two main subcategories: hypo- and hyperkinetic with posture affected in 40/60 hypokinetic and 4/44 hyperkinetic moving fetuses. The majority of the disorders resulted in an adverse outcome. Fourteen percent survived with a handicap, depending on the underlying disorder. The 16 disorders with hypokinetic motility had mainly an autosomal recessive etiology with no possibility of invasive prenatal diagnosis or conclusive sonographic structural anomalies, in contrast to the 17 disorders with hyperkinetic motility. W ithin the limitations of the studies, a deeper understanding of affected milestones in motor devel opment can be obtained. The author concluded that broadening motor assessment procedures from quantitative to quali tative aspects, including the assessment of behavioral states and emphasizing onset and continuity of motility before and after birth will enhance the reliability and predictive val ue of motility as a parameter in the assessment of fetal condition. ${ }^{49}$

A hmed and his group established a behavioral state profile in the eight high risk fetuses by 4D US recording..$^{50}$ In the study the authors tried to observe all the behavioral parameters which are related to CNS function. In anencephalic case and cephalocel e case, they noticed excessive hypertonic movements, whil e fetuses affected by homocystinuria and $M$ eckel- Grubber syndrome demonstrated hypotonia. The sequence of occurrence of behavioral parameters was clearly abnormal in those fetuses affected by anencephalic and cephalocele. These findings fully agree with their previous case report on the behavior of an anencephalic fetus. ${ }^{51} \mathrm{~N}$ ormal quantity and quality of behavioral parameters was noted in fetuses affected by prune belly, diaphragmatic hernia, and hydrothorax. The quality and quantity of fetal movement in the fetus affected by anchondrogenesis were normal, but it was difficult to observe the movement of extremities due to abnormal fetal posture.

From previously described case report, fetal hand movements in the anencephalic fetus were visible only in one direction (hand to head) and it appeared abnormal and monotonous. ${ }^{51}$ I solated arm movements and isolated leg movements were present but the characteristic of movements showed a forceful quality, they were jerky and appeared incidentally. Decreased variability was observed al ong with the tendency of movement patterns appearing randomly compared to being sporadic and continuous in a normal fetus. A Ithough it was difficult to establish the movement patterns in the anencephalic fetus, these results proposed that there was a prominent underlying structure. $\mathrm{H}$ ands occurred around the specific body part and were directed against the uterine wall. W ith the mal formation of fetal CNS, movement's patterns were abnormal and could exist in spite of a serious reduction in the quantity and change in the fetal CNS. ${ }^{51}$

\section{Antenatal Behavior Screening}

Despite the long-standing belief that it is possible to make valid assessment about brain function from observed, no general ized behavior screening method has been developed to identify fetuses that may have central nervous system defects. In the recent study from Morokuma the effort has been made to produce screening test that would be less time consuming and in that way cost effective as compared to their previous study. ${ }^{52}$ They devised a brief ultrasound examination to distinguish fetuses with compromised central nervous system function from the general population and evaluated it within their study. ${ }^{53}$ The study design compared findings on five behavioral patterns obtained by retrospectively reviewing the ultrasound examinations of 5 fetuses who had abnormal behavior with prospectively obtained findings of 29 normal fetuses. M edian time for brief examination criteria was $50 \mathrm{~min}$ (range, 30-60 min) with the only case undetectable by this brief ultrasound examination had an eye-movement period significantly longer than the normal upper limit. The assessment of fetal brain function as part of routine antenatal care could be obtained using this method as a screening test although the disadvantage is that is very time consuming. ${ }^{53}$

In the recent study the Zagreb group attempted to produce a new scoring system for fetal neurobehavior based on prenatal assessment by $3 \mathrm{D} / 4 \mathrm{D}$ sonography. ${ }^{54}$ Parameters that were analyzed were the product of multicentric studies conducted during several years which resulted with the most significant parameters for the assessment of fetal neurological development. There is a similarity between neonatal optimality 
test of A miel-Tison and that new scoring system for the assessment of neurological status in fetuses. ${ }^{55,56}$ One of the differences was that the analytical criteria of typical passive and active tone in the neonate cannot be elicited in the fetus: head anteflexion versus retroflexion, ventral versus dorsal incurvations in the axis, both being of the utmost importance postnatally to confirm CNS optimality. ${ }^{56}$

To produce the new scoring test the Zagreb group identified severely brain damaged infants and those with optimal neurological findings by comparing fetal with neonatal findings. In the group of 100 low-risk pregnancies they retrospectively applied new scoring system. After delivery, postnatal neurological assessment (ATNAT) was performed, ${ }^{70}$ and all neonates assessed as normal reached a score between 14 and 20 , which was assumed to be a score of optimal neurological development. $\mathrm{N}$ ew scoring system was applied in the group of 120 high risk pregnancies in which, based on postnatal neurological findings, three subgroups of newborns were found: normal, mildly or moderately abnormal and abnormal. B ased on this, a neurological scoring system has been proposed. All normal fetuses reached a score in the range from 14 to 20 . Ten fetuses who were postnatally described as mildly or moderately abnormal achieved prenatal score of 5 to 13 prenatally, while another ten fetuses postnatally assigned as neurologically abnormal had a prenatal score from 0-5. A mong this group four had alobar holoprosencephally, one had severe hypertensive hydrocephaly, one had tanatophoric dysplasia and four fetuses had multiple malformations.

That was a preliminary study which is planed to be continued in several collaborative centers. It is hoped that the future database formed using this new score for fetal neurological assessment will help in distinguishing fetal brain and neurodevelopmental alternations due to the early brain impairment occurring inutero. Study of a large population will hopefully validate the value of the new test as a predictive marker for fetal neurodevelopmental outcome in both low and high-risk populations.

\section{REFERENCES}

1. Prechtl HFR. Qualitative changes of spontaneous movements in fetus and preterm infant are a marker of neurological dysfunction. Early Hum Dev 1990;23:151-58.

2. Nijhuis J G (Ed): Fetal B ehaviour: Developmental and Perinatal A spects. Oxford: Oxford U niversity Press, 1992.

3. A hmed B, Kurjak A, A ndonotopo W, K henyab N, Saleh N, A I$M$ ansoori Z. Fetal behavioral and structural abnormalities in high-risk fetuses assessed by 4D sonography. Ultrasound Rev Obstet Gynecol 2005;5:275-87.

4. Kurjak A, M iskovic B, Stanojevic M, A miel-Tison C, A hmed $B$, Azumendi $G, V$ asilj $O$, et al. New scoring system for fetal neurobehavior assessed by three- and four-dimensional sonography. J Perinat Med 2008;36(1):73-81.
5. Strijbis EM M, Oudman I, van Essen $P, M$ acLennan A H. Cerebral palsy and the application of criteria for acute intrapartum hypoxia. Obstet Gynecol 2006;107:1357-65.

6. V isser GH, B ekedam DJ, M ulder EJ, van Ballegooie E. Delayed emergence of fetal behaviour in type-1 diabetic women. Early Hum Dev 1985;12(2):167-72.

7. M ulder EJ, V isser GH, B ekedam DJ, Prechtl HF. Emergence of behavioural states in fetuses of type-1-diabetic women. Early Hum Dev 1987;15(4):231-51.

8. Nijhuis J G, Prechtl HF, Martin CB J r, B ots RS. A re there behavioural states in the human fetuses? Early Hum Dev 1982:6(2):177-95.

9. Visser GH, M ulder EJ , B ekedam DJ , van B allegooie E, Prechtl HF. Fetal behaviour in type-1 diabetic women. Eur J Obstet Gynecol Reprod Biol. 1986;21(5-6):315-20.

10. M ulder EJ, V isser GH. Growth and motor devel opment in fetuses of women with type-1 diabetes. II. Emergence of specific movement patterns. Early Hum Dev 1991;25(2):107-15.

11. M ulder EJ, Visser GH, M orssink LP, de V ries JI. Growth and motor development in fetuses of women with type- 1 diabetes. III. First trimester quantity of fetal movement patterns. Early Hum Dev 1991;25(2):117-33.

12. M ulder $E J, O$ 'B Bien MJ, L ems $Y L$, V isser GH, Prechtl HF. B ody and breathing movements in near-term fetuses and newborn infants of type-1 diabetic women.Early Hum Dev 1990; 24(2):131-52.

13. Mulder EJ, Visser GH. Impact of early growth delay on subsequent fetal growth and functional development: a study on diabetic pregnancy. Early Hum Dev 1992;31(2):91-95.

14. M ulder EJ, L eiblum D M , V isser GH F etal breathing movements in late diabetic pregnancy: relationship to fetal heart rate patterns and Braxton Hicks' contractions. Early Hum Dev 1995; 24;43(3):225-32.

15. Reece EA, Hagay Z, Roberts A B, D eGennaro N, Homko CJ, Connolly-Diamond $M$, Sherwin R, et al. Fetal Doppler and behavioral responses during hypoglycemia induced with the insulin clamp technique in pregnant diabetic women. A m J Obstet Gynecol 1995;172(1 Pt 1):151-55.

16. Devoe LD, Y oussef AA, Castillo RA, Croom CS. Fetal biophysical activities in thi rd-trimester pregnancies complicated by diabetes mellitus. A m J Obstet Gynecol 1994;171(2):298303.

17. Kainer F, Prechtl HF, Engele H, Einspieler C. Assessment of the quality of general movements in fetuses and infants of women with type-I diabetes mellitus. Early Hum D ev 1997;24;50(1):1325.

18. van V liet MA, Martin CB Jr, Nijhuis J G, Prechtl HF. The relationship between fetal activity and behavioral states and fetal breathing movements in normal and growth-retarded fetuses. A m J Obstet Gynecol 1985;153(5):582-88.

19. Van V liet MA, M artin CB J r, Nijhaus J G, Prechtl HF. $B$ ehavioural states in growth-retarded human fetuses. Early $\mathrm{H}$ um Dev 1985;12:183-97.

20. A rduini D, Rizzo G, Romanini C, M ancuso S. Computerized analysis of behavioural states in asymmetrical growth retarded fetuses. J Perinat M ed 1988;16(4):357-63. 
21. Rizzo G, A rduini D, Pennestri F, Romanini C, M ancuso S. Fetal behaviour in growth retardation: its relationship to fetal blood flow. Prenat Diagn 1987;7(4):229-38.

22. Sival DA, Visser GH, Prechtl HF. The effect of intrauterine growth retardation on the quality of general movements in the human fetus. Early Hum Dev 1992;28(2):119-32.

23. Sival DA, V isser GH, Prechtl HF. The relationship between the quantity and quality of prenatal movements in pregnancies complicated by intra-uterine growth retardation and premature rupture of the membranes. Early H um Dev 1992;30(3):193-209.

24. Ribbert LS, V isser GH, M ulder EJ, Zonneveld M F, M orssink $L P$. C hanges with time in fetal heart rate variation, movement incidences and haemodynamics in intrauterine grow th retarded fetuses: a longitudinal approach to the assessment of fetal well being. Early Hum Dev 1993;31(3):195-208.

25. V indla S, James DK, Sahota DS, Coppens M. Computerised analysis of behaviour in normal and growth-retarded fetuses. Eur J Obstet Gynecol Reprod Biol 1997;75(2):169-75.

26. B ekedam DJ, M ulder EJ, Snijders RJ, V isser GH. The effects of maternal hyperoxia on fetal breathing movements, body movements and heart rate variation in growth retarded fetuses. Early Hum Dev 1991;27(3):223-32.

27. A ndonotopo $W, K$ urjak $A$. The assessment of fetal behavior of growth restricted fetuses by $4 \mathrm{D}$ sonography. J Perinat Med 2006;34:471-78.

28. Brien JF, Smith GN. Effects of alcohol (ethanol) on the fetus.J Dev Physiol 1991;15(1):21-32.

29. M CL eod W, B rien J , L oomis C, C armichael L, Probert C, Patrick $J$. Effect of maternal ethanol ingestion on fetal breathing movements, gross body movements, and heart rate at 37 to 40 weeks' gestational age. A m J Obstet Gynecol 1983;145(2):25157.

30. Fox HE, Steinbrecher M, Pessel D, Inglis J, M edvid L, Angel E.M aternal ethanol ingestion and the occurrence of human fetal breathing movements. Am J Obstet Gynecol 1978;132(4): 354-58.

31. Mulder EJ, Kamstra A, O'B rien MJ, V isser GH, Prechtl HF. A bnormal fetal behavioural state regulation in a case of high maternal alcohol intake during pregnancy. Early Hum Dev 1986;14(3-4):321-26.

32. Mulder EJ, M orssink LP, van der Schee T, V isser GH. A cute maternal alcohol consumption disrupts behavioral state organization in the near-term fetus. Pediatr Res 1998;44(5): 774-79.

33. Hume RF J r, O'Donnell KJ, Stanger CL, K illam A P, Gingras $J \mathrm{~L}$. In utero cocaine exposure: observations of fetal behavioral state may predict neonatal outcome. A m J Obstet Gynecol 1989;161(3):685-90.

34. Hepper PG Human fetal behaviour and maternal cocaine use: a longitudinal study. Neurotoxicology 1995 Spring;16(1):139-43.

35. Gingras J L, O'Donnell KJ. State control in the substanceexposed fetus. I. The fetal neurobehavioral profile: an assessment of fetal state, arousal, and regulation competency. A nn N Y A cad Sci 1998;846:262-76.
36. Jansson LM, Dipietro J, Elko A. Fetal response to maternal methadone administration.Am J Obstet Gynecol 2005;193 (3 Pt 1):611-17.

37. Radetti G, Zavallone A, Gentili L, Beck-Peccoz P, Bona G. Foetal and neonatal thyroid disorders. Minerva Pediatr 2002;54(5):383-400.

38. Serup J, Petersen S. Hyperthyroidism during pregnancy treated with propylthiouracil. The significance of maternal and foetal parameters. A cta Obstet Gynecol Scand 1977;56(5):463-66.

39. V intzileos A M , Campbell WA, Nochimson DJ, Connolly ME, Fuenfer M M , Hoehn GJ Thefetal biophysical profile in patients with premature rupture of the membranes- an early predictor of fetal infection. A m J Obstet Gynecol 1985;152(5):510-16.

40. Konstantinidou A, A nninos H, Spanakis N , K otsiakis X, Syridou $G$, Tsakris A, Patsouris E. Transplacental infection of Coxsackievirus B3 pathological findings in the fetus. J Med Virol 2007;79(6):754-57.

41. Craig S, Permezel M, Doyle L, M ildenhall L, Garland S. Perinatal infection with Listeria monocytogenes. A ust NZJ Obstet Gynaecol 1996;36(3):286-90.

42. Goldstein I, Romero R, M errill S, W an M , O'Connor TZ, M azor $M$, Hobbins JC. Fetal body and breathing movements as predictors of intraamniotic infection in preterm premature rupture of membranes. A m J Obstet Gynecol 1988;159(2):363-68.

43. Del Valle GO, J offe GM , Izquierdo LA, Smith JF, Gilson GJ, Curet $L B$. The biophysical profile and the nonstress test: poor predictors of chorioamnionitis and fetal infection in prolonged preterm premature rupture of membranes. Obstet Gynecol 1992;80(1):106-10.

44. Sherer DM, Spong CY, M inior VK, Salafia CM. Decreased amniotic fluid volume at $<32$ weeks of gestation is associated with decreased fetal movements. Am J Perinatol 1996;13(8): 479-82.

45. Habek D Effects of smoking and fetal hypokinesia in early pregnancy. A rch M ed Res 2007;38(8):864-67.

46. Cowperthwaite $B$, Hains SM, K isilevsky BS. Fetal behavior in smoking compared to non-smoking pregnant women. Infant Behav Dev 2007;30(3):422-30 Epub 2007 12.Links.

47. Warner J, Hains SM, K isilevsky BS. A n exploratory study of fetal behavior at 33 and 36 w eeks gestational age in hypertensive women. Dev Psychobiol 2002;41(2):156-68.

48. K isilevsky BS, Hains SM. C omparison of fetal behavior in lowand high-risk pregnancies. Fetal Pediatr Pathol 2005;24(1):120.

49. de V ries JI, Fong BF. Changes in fetal motility as a result of congenital disorders: an overview. Ultrasound Obstet Gynecol 2007;29:590-99.

50. A hmed B, Kurjak A, A ndonotopo W, K henyab N, Saleh N, A I$M$ ansoori Z. Fetal behavioral and structural abnormalities in high risk fetuses assessed by 4D sonography. The UItrasound Review of Obstetrics and Gynecology 2005;5:1-13.

51. A ndonotopo W, A Kurjak, MI Kosuta: B ehavioral of anencephalic fetus studied by 4D sonography. J M atern Fetal Neonatal M ed 2005;17:165. 
52. Horimoto $\mathrm{N}$, Koyanagi $\mathrm{T}, \mathrm{M}$ aeda $\mathrm{H}$, Satoh $\mathrm{S}$, Takashima $\mathrm{T}$, $\mathrm{M}$ inami T, Nakano H. Can brain impairment be detected by in utero behavioural patterns? Arch Dis Child 1993;69:3-8.

53. M orokuma S, Fukushima K, Y umoto $Y, U$ chimura $M$, Fujiwara $A, M$ atsumoto $M$, Satoh $S$, et al. Simplified ultrasound screening for fetal brain function based on behavioral pattern. Early Hum Dev 2007;83:177-81.

54. Kurjak A, M iskovic B, Stanojevic $M$, A miel-Tison C, A hmed $B$, A zumendi $G, V$ asilj $O$, et al. New scoring system for fetal neurobehavior assessed by three- and four-dimensional sonography. J Perinat M ed 2008;36(1):73-81.

55. A miel-Tison C. Neurological assessment of the neonate revisited: a personal view. Dev Med Child Neurol 1990;32: 1105-13.

56. A miel-Tison C, Gosselin J, Kurjak A. Neurosonography in the second half of fetal life: a neonatologist's point of view. J Perinat M ed 2006;34:437-46. 